\title{
Properties of convolutions for hypergeometric series with univalent functions
}

\section{Ji A Kim and Nak Eun Cho*}

\section{"Correspondence:} necho@pknu.ac.kr Department of Applied

Mathematics, Pukyong National

University, Busan, 608-737, Korea

\begin{abstract}
The purpose of the present paper is to investigate various mapping and inclusion properties involving subclasses of analytic and univalent functions for a linear operator defined by means of Hadamard product (or convolution) with the Gaussian hypergeometric function.

MSC: $30 C 45 ; 30 C 55 ; 33 C 20$

Keywords: univalent function; starlike function; convex function; uniformly convex; uniformly starlike; hypergeometric function
\end{abstract}

\section{Introduction}

Let $\mathcal{A}$ denote the class of functions of the form

$$
f(z)=z+\sum_{n=2}^{\infty} a_{n} z^{n}
$$

which are analytic in the open unit disk $\mathbb{U}=\{z \in \mathbb{C}:|z|<1\}$. Denote by $\mathcal{S}$ the class of all functions in $\mathcal{A}$ which are univalent in $\mathbb{U}$.

A function $f \in \mathcal{A}$ is said to be in the class $\mathcal{R}^{t}(A, B)$ if

$$
\left|\frac{f^{\prime}(z)-1}{t(A-B)-B\left(f^{\prime}(z)-1\right)}\right|<1 \quad(-1 \leq B<A \leq 1 ; t \in \mathbb{C} \backslash\{0\} ; z \in \mathbb{U}),
$$

Clearly, a function $f$ belongs to $\mathcal{R}^{t}(A, B)$ if and only if there exists a function $w$ regular in $\mathbb{U}$ satisfying $w(0)=0$ and $|w(z)|<1(z \in \mathbb{U})$ such that

$$
1+\frac{1}{t}\left(f^{\prime}(z)-1\right)=\frac{1+A w(z)}{1+B w(z)} \quad(z \in \mathbb{U})
$$

The class $\mathcal{R}^{t}(A, B)$ was introduced by Dixit and Pal [1]. By giving specific values to $t, A$ and $B$ in (1.2), we obtain the following subclasses studied by various researchers in earlier works:

( 2013 Kim and Cho; licensee Springer. This is an Open Access article distributed under the terms of the Creative Commons Attribution License (http://creativecommons.org/licenses/by/2.0), which permits unrestricted use, distribution, and reproduction in any medium, provided the original work is properly cited. 
(i) For $t=e^{-i \eta} \cos \eta(|\eta|<\pi / 2), A=1-2 \alpha(0 \leq \alpha<1)$ and $B=-1$, we obtain the class of functions $f$ satisfying the condition:

$$
\left|\frac{e^{i \eta}\left(f^{\prime}(z)-1\right)}{2(1-\alpha) \cos \eta+e^{i \eta}\left(f^{\prime}(z)-1\right)}\right|<1 \quad(z \in U) .
$$

In this case, the class $\mathcal{R}^{t}(A, B)$ is equivalent to the class $\mathcal{R}_{\eta}(\alpha)$ which is studied by Ponnusamy and Rønning [2]. Here, $\mathcal{R}_{\eta}(\alpha)$ is the class of functions $f \in \mathcal{A}$ satisfying the condition:

$$
\operatorname{Re}\left(e^{i \eta}\left(f^{\prime}(z)-\alpha\right)\right)>0 \quad(|\eta|<\pi / 2 ; 0 \leq \alpha<1 ; z \in \mathbb{U}) .
$$

(ii) For $t=e^{i \eta} \cos \eta(|\eta|<\pi / 2)$, we obtain the class of functions $f \in \mathcal{A}$ satisfying the condition

$$
\left|\frac{e^{i \eta}\left(f^{\prime}(z)-1\right)}{B e^{i \eta} f^{\prime}(z)-(A \cos \eta+i B \sin \eta)}\right|<1 \quad(z \in \mathbb{U}),
$$

which was studied by Dashrath [3].

(iii) For $t=1, A=\beta$ and $B=-\beta(0<\beta \leq 1)$, we obtain the class of functions $f$ satisfying the condition:

$$
\left|\frac{f^{\prime}(z)-1}{f^{\prime}(z)+1}\right|<\beta \quad(0<\beta \leq 1 ; z \in \mathbb{U})
$$

which was studied by Caplinger and Cauchy [4] and Padmanabhan [5].

Let $\mathcal{S}^{*}(\alpha)$ and $\mathcal{C}(\alpha)$ denote the subclasses of $\mathcal{A}$ consisting of starlike and convex functions of order $\alpha(0 \leq \alpha<1)$ in $\mathbb{U}$, respectively. It is well known that $\mathcal{S}^{*}(\alpha) \subset \mathcal{S}^{*}(0) \equiv \mathcal{S}^{*}, \mathcal{C}(\alpha) \subset$ $\mathcal{C}(0) \equiv \mathcal{C}$ and $\mathcal{K}(\alpha) \subset \mathcal{S}^{*}(\alpha) \subset \mathcal{S}$. For $\lambda>0$, define

$$
\mathcal{S}_{\lambda}^{*}=\left\{f \in \mathcal{A}:\left|\frac{z f^{\prime}(z)}{f(z)}-1\right|<\lambda, z \in \mathbb{U}\right\}
$$

and

$$
\mathcal{C}_{\lambda}=\left\{f \in \mathcal{A}: z f^{\prime}(z) \in \mathcal{S}_{\lambda}^{*}\right\}
$$

It is a known fact that a sufficient condition for $f \in \mathcal{A}$ of the form (1.1) to belong to the class $\mathcal{S}^{*}$ is that $\sum_{n=2}^{\infty} n a_{n} \leq 1$. A simple extension of this result is the following [6]:

$$
\sum_{n=2}^{\infty}(n+\lambda-1)\left|a_{n}\right| \leq \lambda \quad \Longrightarrow \quad f \in \mathcal{S}_{\lambda}^{*} .
$$

For $\lambda=1 / 2$, this was previously proved by Schild [2]. Since $f \in \mathcal{C}_{\lambda}$ if and only if $z f^{\prime}(z) \in \mathcal{S}_{\lambda}^{*}$, we have a corresponding results for $\mathcal{C}_{\lambda}$,

$$
\sum_{n=2}^{\infty} n(n+\lambda-1)\left|a_{n}\right| \leq \lambda \quad \Longrightarrow \quad f \in \mathcal{C}_{\lambda} .
$$


Now we introduce the class $\mathcal{U S T}$ (resp., $\mathcal{U C V}$ ) of uniformly starlike (resp., convex) functions. We say $[7,8]$ that $f \in \mathcal{A}$ is in $\mathcal{U S T}$ (resp., $\mathcal{U C V}$ ) if for each $\xi \in \mathbb{U}$ and each circular $\operatorname{arc} \gamma$ in $\mathbb{U}$ with center $\eta$, the image $\operatorname{arc} f(\gamma)$ is starlike with respect to $f(\xi)$ (resp., is a convex curve).

In this paper, we consider the Gaussian hypergeometric function $F(a, b ; c ; z)$ defined by

$$
F(a, b ; c ; z)=\sum_{n=0}^{\infty} \frac{(a)_{n}(b)_{n}}{(c)_{n}} \frac{z^{n}}{n !} \quad(a, b \in \mathbb{C} ; c \neq 0,-1,-2, \ldots ; z \in \mathbb{U})
$$

where $(v)_{n}$ is the Pochhammer symbol (or the shifted factorial) defined (in terms of the Gamma function) by

$$
(v)_{n}:=\frac{\Gamma(v+n)}{\Gamma(v)}= \begin{cases}1 & \text { if } n=0 \text { and } v \in \mathbb{C} \backslash\{0\} \\ v(v+1) \cdots(v+n-1) & \text { if } n \in \mathbb{N} \text { and } v \in \mathbb{C}\end{cases}
$$

We note that $F(a, b ; c ; z)=F(b, a ; c ; z)$ and

$$
F(a, b ; c ; 1)=\frac{\Gamma(c-a-b) \Gamma(c)}{\Gamma(c-a) \Gamma(c-b)} \quad(\operatorname{Re}(c-a-b)>0) .
$$

We also recall (see [4]) that the function $F(a, b ; c ; z)$ is bounded if $\operatorname{Re}\{c-a-b\}>0$, and has a pole at $z=1$ if $\operatorname{Re}\{c-a-b\} \leq 0$. Moreover, univalence, starlikeness and convexity properties of $z F(a, b ; c ; z)$ have been studied extensively in Ponnusamy and Vuorinen [9] and Ruscheweyh and Singh [10].

For $f \in \mathcal{A}$, we define the operator $I_{a, b ; c} f$ by

$$
I_{a, b ; c} f(z)=z F(a, b ; c ; z) * f(z)
$$

where $*$ denotes the usual Hadamard product (or convolution) of power series. If $f$ equals to the convex function $z /(1-z)$, then the operator $I_{a, b ; c} f(z)$ becomes $z F(a, b ; c ; z)$. For a survey of special cases of this operator and also more general operators, we can refer to the article by Srivastava [11-13] and Swaminathan [14], where also a long list of other references can be found. Thus, the operator $I_{a, b ; c} f$ and hence the Gaussian hypergeometric function is a natural object for studying inclusion properties related to the convolution product. In the present paper, we find a condition for univalency of the operator $I_{a, b ; c} f$. We also investigate conditions such that $I_{a, b ; f} f \in \mathcal{R}^{t}(A, B)\left(\mathcal{U S T}, \mathcal{U C V}, \mathcal{S}_{\lambda}^{*}\right.$ and $\left.\mathcal{C}_{\lambda}\right)$, whenever $f \in \mathcal{R}^{t}(A, B)$.

\section{A set of lemmas}

Now we introduce several lemmas which are needed for the proof of our main results.

Lemma 2.1 [1] Let a function $f$ of the form (1.1) be in $\mathcal{R}^{t}(A, B)$. Then

$$
\left|a_{n}\right| \leq \frac{(A-B)|t|}{n}
$$

The result is sharp for the function

$$
f(z)=\int_{0}^{z}\left(1+\frac{(A-B) t z^{n-1}}{1+B z^{n-1}}\right) d z \quad(n \geq 2 ; z \in \mathbb{U}) .
$$


Lemma 2.2 [1] Let a function $f$ of the form (1.1) be in $\mathcal{A}$. If

$$
\sum_{n=2}^{\infty}(1+|B|) n\left|a_{n}\right| \leq(A-B)|t| \quad(-1 \leq B<A \leq 1 ; t \in \mathbb{C})
$$

then $f \in \mathcal{R}^{t}(A, B)$. The result is sharp for the function

$$
f(z)=z+\frac{(A-B) t}{(1+|B|) n} z^{n} \quad(n \geq 2 ; z \in \mathbb{U}) .
$$

Lemma 2.3 [15] Let $w(z)$ be regular in the unit disk $\mathbb{U}$ with $w(0)=0$. Then, if $|w(z)|$ attains a maximum value on the circle $|z|=r(0 \leq r<1)$ at a point $z$, we can write

$$
z_{1} w^{\prime}\left(z_{1}\right)=m w\left(z_{1}\right)
$$

where $m$ is real and $m \geq 1$.

Lemma 2.4 [2] (i) For $a, b \in \mathbb{C} \backslash\{0,1\}$ and $c \in \mathbb{C} \backslash\{1\}$ with $c>\max \{0, a+b-1\}$,

$$
\sum_{n=0}^{\infty} \frac{(a)_{n}(b)_{n}}{(c)_{n}(1)_{n+1}}=\frac{1}{(a-1)(b-1)}\left(\frac{\Gamma(c+1-a-b) \Gamma(c)}{\Gamma(c-a) \Gamma(c-b)}-(c-1)\right) .
$$

(ii) For $a, b \in \mathbb{C} \backslash\{0\}$ with $a>0$ and $b>0$ and $c>a+b+1$,

$$
\sum_{n=0}^{\infty} \frac{(n+1)(a)_{n}(b)_{n}}{(c)_{n}(1)_{n}}=\left(\frac{a b}{c-a-b-1}+1\right) \frac{\Gamma(c-a-b) \Gamma(c)}{\Gamma(c-a) \Gamma(c-b)} .
$$

Lemma 2.5 [16] A function $f$ of the form (1.1) is in $\mathcal{U C V}$ if

$$
\sum_{n=2}^{\infty} n(2 n-1)\left|a_{n}\right| \leq 1
$$

Lemma 2.6 [16] A function $f$ of the form (1.1) is in $\mathcal{U S T}$ if

$$
\sum_{n=2}^{\infty}(3 n-2)\left|a_{n}\right| \leq 1
$$

\section{Main results}

Theorem 3.1 Let $f \in \mathcal{A}$. If

$$
\left|\left(I_{a, b ; f} f(z)\right)^{\prime}-1\right|^{1-\beta}\left|\frac{z\left(I_{a, b ; c} f(z)\right)^{\prime \prime}}{\left(I_{a, b ; c} f(z)\right)^{\prime}}\right|^{\beta}<\frac{1}{2^{\beta}} \quad(\beta \geq 0),
$$

then $I_{a, b ;} f$ is univalent in $\mathbb{U}$.

Proof We note that

$$
I_{a, b ; c}(f)=z+\sum_{n=2}^{\infty} \frac{(a)_{n-1}(b)_{n-1}}{(c)_{n-1}(1)_{n-1}} a_{n} z^{n}
$$


in $\mathcal{A}$. Define $w$ by

$$
w(z)=\left(I_{a, b ; c} f(z)\right)^{\prime}-1
$$

for $z \in \mathbb{U}$. Then it follows that $w$ is analytic in $\mathbb{U}$ with $w(0)=0$. By (3.1),

$$
\begin{aligned}
& |w(z)|^{1-\beta}\left|\frac{z w^{\prime}(z)}{1+w(z)}\right|^{\beta} \\
& \quad=|w(z)|\left|\frac{z w^{\prime}(z)}{w(z)} \frac{1}{1+w(z)}\right|^{\beta}<\frac{1}{2^{\beta}} .
\end{aligned}
$$

Suppose that there exists a point $z_{1} \in \mathbb{U}$ such that

$$
\max _{|z| \leq\left|z_{1}\right|}|w(z)|=\left|w\left(z_{1}\right)\right|=1
$$

Then, by Lemma 2.3, we can put

$$
\frac{z_{1} w^{\prime}\left(z_{1}\right)}{w\left(z_{1}\right)}=m \geq 1
$$

Therefore, we obtain

$$
\left|w\left(z_{1}\right)\right|\left|\frac{z_{1} w^{\prime}\left(z_{1}\right)}{w\left(z_{1}\right)} \frac{1}{1+w(z)}\right|^{\beta} \geq\left(\frac{m}{2}\right)^{\beta} \geq \frac{1}{2^{\beta}},
$$

which contradicts the condition (3.2). This shows that

$$
|w(z)|=\left|\left(I_{a, b ; c} f(z)\right)^{\prime}-1\right|<1,
$$

which implies that $\operatorname{Re}\left(I_{a, b ; c} f(z)\right)^{\prime}>0$ for $z \in \mathbb{U}$. Therefore, by the Noshiro-Warschawski theorem [17], $I_{a, b ; c} f$ is univalent in $\mathbb{U}$.

Theorem 3.2 Let $a, b \in \mathbb{C} \backslash\{0\}$ and $c>|a|+|b|$. Suppose that $f \in \mathcal{R}^{t}(A, B)$ and satisfy the condition

$$
\frac{\Gamma(c-|a|-|b|) \Gamma(c)}{\Gamma(c-|a|) \Gamma(c-|b|)} \leq \frac{1}{1+|B|}+1
$$

Then the operator $I_{a, b ; c} f$ maps $\mathcal{R}^{t}(A, B)$ into $\mathcal{R}^{t}(A, B)$.

Proof Let $a, b \in \mathbb{C} \backslash\{0\}, c>|a|+|b|$ and suppose that $f(z)=z+\sum_{n=2}^{\infty} a_{n} z^{n} \in \mathcal{R}^{t}(A, B)$. Then, by Lemma 2.2 , it suffices to show that

$$
T_{1}:=\sum_{n=2}^{\infty}(1+|B|) n\left|A_{n}\right| \leq(A-B)|t|,
$$

where

$$
A_{n}=\frac{(a)_{n-1}(b)_{n-1}}{(c)_{n-1}(1)_{n-1}} a_{n} .
$$


From Lemma 2.1 and the fact that $\left|(a)_{n}\right| \leq(|a|)_{n}$, we have

$$
\begin{aligned}
T_{1} \leq & \sum_{n=2}^{\infty}(A-B)|t|(1+|B|) \frac{(|a|)_{n-1}(|b|)_{n-1}}{(c)_{n-1}(1)_{n-1}} \\
& \times(A-B)|t|(1+|B|)\left(\sum_{n=0}^{\infty} \frac{(|a|)_{n}(|b|)_{n}}{(c)_{n}(1)_{n}}-1\right) .
\end{aligned}
$$

Using the formula (1.7) and the assumption, we find that

$$
\begin{aligned}
T_{1} & \leq(A-B)|t|(1+|B|)\left(\frac{\Gamma(c-|a|-|b|) \Gamma(c)}{\Gamma(c-|a|) \Gamma(c-|b|)}-1\right) \\
& \leq(A-B)|t|,
\end{aligned}
$$

which implies that the operator $I_{a, b ; c} f$ maps $\mathcal{R}^{t}(A, B)$ into $\mathcal{R}^{t}(A, B)$.

If, in the proof of Theorem 3.2, we take $b=\bar{a}$, then we have the following theorem under a weaker condition on the parameter $c$.

Theorem 3.3 Let $a \in \mathbb{C} \backslash\{0\}$ and $c>2 \operatorname{Re}\{a\}$. Suppose that $f \in \mathcal{R}^{t}(A, B)$ and satisfy the condition

$$
\frac{\Gamma(c-2 \operatorname{Re} a) \Gamma(c)}{\Gamma(c-a) \Gamma(c-\bar{a})} \leq \frac{1}{1+|B|}+1
$$

Then the operator $I_{a, \bar{a} ; c}(f)$ maps $\mathcal{R}^{t}(A, B)$ into $\mathcal{R}^{t}(A, B)$.

Proof The proof of Theorem 3.3 follows in the similar lines on the proof of Theorem 3.2 and so we omit the details.

Theorem 3.4 Let $a, b \in \mathbb{C} \backslash\{0\}$ and $\lambda \in(0,1]$. Suppose that $f \in \mathcal{R}^{t}(A, B),|a| \neq 1,|b| \neq 1$ and $c \neq 1$ such that $c>|a|+|b|$ and satisfy the condition

$$
\begin{gathered}
\frac{\Gamma(c-|a|-|b|) \Gamma(c)}{\Gamma(c-|a|) \Gamma(c-|b|)}\left(1+\frac{(\lambda-1)(c-|a|-|b|)}{(|a|-1)(|b|-1)}\right) \\
\quad \leq \lambda\left(1+\frac{1}{(A-B)|t|}\right)+\frac{(\lambda-1)(c-1)}{(|a|-1)(|b|-1)} .
\end{gathered}
$$

Then the operator $I_{a, b ; c} f$ maps $\mathcal{R}^{t}(A, B)$ into $\mathcal{S}_{\lambda}^{*}$.

Proof Let $a, b \in \mathbb{C} \backslash\{0\}$ and $c>|a|+|b|$ with $|a| \neq 1,|b| \neq 1$ and $c \neq 1$. Suppose that $f(z)=$ $z+\sum_{n=2}^{\infty} a_{n} z^{n} \in \mathcal{R}^{t}(A, B)$. Then, by (1.5), it is sufficient to show that

$$
T_{2}:=\sum_{n=2}^{\infty}(n+\lambda-1)\left|\frac{(a)_{n-1}(b)_{n-1}}{(c)_{n-1}(1)_{n-1}} a_{n}\right| \leq \lambda .
$$


By using Lemma 2.1 and (i) of Lemma 2.4, we observe that

$$
\begin{aligned}
T_{2} \leq & \sum_{n=2}^{\infty}(n+\lambda-1) \frac{(|a|)_{n-1}(|b|)_{n-1}}{(c)_{n-1}(1)_{n-1}} \frac{(A-B)|t|}{n} \\
= & (A-B)|t|\left[\sum_{n=1}^{\infty} \frac{(|a|)_{n}(|b|)_{n}}{(c)_{n}(1)_{n}}+(\lambda-1) \sum_{n=1}^{\infty} \frac{(|a|)_{n}(|b|)_{n}}{(c)_{n}(1)_{n+1}}\right] \\
= & (A-B)|t|\left[\left(\frac{\Gamma(c-|a|-|b|) \Gamma(c)}{\Gamma(c-|a|) \Gamma(c-|b|)}-1\right)\right. \\
& \left.+(\lambda-1)\left\{\frac{1}{(|a|-1)(|b|-1)}\left(\frac{\Gamma(c+1-|a|-|b|) \Gamma(c)}{\Gamma(c-|a|) \Gamma(c-|b|)}-(c-1)\right)-1\right\}\right] \\
= & (A-B)|t|\left[\frac{\Gamma(c-|a|-|b|) \Gamma(c)}{\Gamma(c-|a|) \Gamma(c-|b|)}\left(1+\frac{(\lambda-1)(c-|a|-|b|)}{(|a|-1)(|b|-1)}\right)\right. \\
& \left.-\frac{(\lambda-1)(c-1)}{(|a|-1)(|b|-1)}-\lambda\right] \leq \lambda,
\end{aligned}
$$

by (3.5), which completes the proof of Theorem 3.4.

Taking $\lambda=1$ and $b=\bar{a}$ in Theorem 3.4, we have the following result.

Corollary3.1 Let $a \in \mathbb{C} \backslash\{0\}$ and $c>\max \{0,2 \operatorname{Re}\{a\}\}$. Suppose that $f \in \mathcal{R}^{t}(A, B)$ and satisfy the condition

$$
\frac{\Gamma(c-2 \operatorname{Re}\{a\}) \Gamma(c)}{\Gamma(c-a) \Gamma(c-\bar{a})} \leq 1+\frac{1}{(A-B)|t|} .
$$

Then $I_{a, \bar{a} ; c} f \in \mathcal{S}_{1}^{*}$.

By using the same method as in the proof of Theorem 3.4, we have the following result.

Theorem 3.5 Let $a, b \in \mathbb{C} \backslash\{0\}$ and $c>1+|a|+|b|, \lambda \in(0,1]$ and $f \in \mathcal{R}^{t}(A, B)$. Suppose that

$$
\frac{\Gamma(c-|a|-|b|)}{\Gamma(c-|a|) \Gamma(c-|b|)}\left(\frac{|a b|}{c-|a|-|b|-1}+\lambda\right) \leq \lambda\left(1+\frac{1}{(A-B)|t|}\right) .
$$

Then the operator $I_{a, b ; f} f$ maps $\mathcal{R}^{t}(A, B)$ into $\mathcal{C}_{\lambda}$

Proof Let $a, b \in \mathbb{C} \backslash\{0\}$ and $c>|a|+|b|+1$. Suppose $f(z)=z+\sum_{n=2}^{\infty} a_{n} z^{n} \in \mathcal{R}^{t}(A, B)$. To show that the operator $I_{a, b ; c} f$ belongs to $\mathcal{C}_{\lambda}$, from (1.6), it is enough to show that

$$
T_{3}:=\sum_{n=2}^{\infty} n(n+\lambda-1)\left|\frac{(a)_{n-1}(b)_{n-1}}{(c)_{n-1}(1)_{n-1}} a_{n}\right| \leq \lambda .
$$


From Lemma 2.1 and (1.7), we find that

$$
\begin{aligned}
T_{3} & \leq(A-B)|t|\left[\sum_{n=1}^{\infty} \frac{(|a|)_{n}(|b|)_{n}}{(c)_{n}(1)_{n-1}}+\lambda \sum_{n=1}^{\infty} \frac{(|a|)_{n}(|b|)_{n}}{(c)_{n}(1)_{n}}\right] \\
& =(A-B)|t|\left[\frac{|a b|}{c} \sum_{n=0}^{\infty} \frac{(|a|+1)_{n}(|b|+1)_{n}}{(c+1)_{n}(1)_{n}}+\lambda \sum_{n=1}^{\infty} \frac{(|a|)_{n}(|b|)_{n}}{(c)_{n}(1)_{n}}\right] \\
& =(A-B)|t|\left[\frac{|a b|}{c} \frac{\Gamma(c-|a|-|b|-1) \Gamma(c+1)}{\Gamma(c-|a|) \Gamma(c-|b|)}+\lambda \frac{\Gamma(c-|a|-|b|) \Gamma(c)}{\Gamma(c-|a|) \Gamma(c-|b|)}\right] \\
& =(A-B)|t|\left[\frac{\Gamma(c-|a|-|b|) \Gamma(c)}{\Gamma(c-|a|) \Gamma(c-|b|)}\left(\frac{|a b|}{c-|a|-|b|-1}+\lambda\right)-\lambda\right] \\
& \leq \lambda,
\end{aligned}
$$

by (3.6) and the conclusion follows.

Similarly, taking $\lambda=1$ and $b=\bar{a}$ in Theorem 3.5 , we have the following result.

Corollary 3.2 Let $a \in \mathbb{C} \backslash\{0\}, c>\max \{0,1+2 \operatorname{Re}\{a\}\}$ and $\lambda \in(0,1]$. Suppose that

$$
\frac{\Gamma(c-2 \operatorname{Re}\{a\}) \Gamma(c)}{\Gamma(c-a) \Gamma(c-\bar{a})}\left(\frac{|a|^{2}}{c-1-2 \operatorname{Re}\{a\}}+1\right) \leq 1+\frac{1}{(A-B)|t|} .
$$

Then $I_{a, \bar{a} ; c} f \in \mathcal{C}_{1}$.

By using Lemma 2.5 and Lemma 2.6, we have the following theorem for $\mathcal{U C V}$ and $\mathcal{U S T}$.

Theorem 3.6 Let $a, b \in \mathbb{C} \backslash\{0\}, c>|a|+|b|+1$ and $f \in \mathcal{R}^{t}(A, B)$. Suppose that

$$
(A-B)|t|\left[\frac{\Gamma(c-|a|-|b|) \Gamma(c)}{\Gamma(c-|a|) \Gamma(c-|b|)}\left(\frac{2|a b|}{c-|a|-|b|-1}+1\right)-1\right] \leq 1 .
$$

Then the operator $I_{a, b ; c} f$ maps $\mathcal{R}^{t}(A, B)$ into $\mathcal{U C V}$.

Proof Let $a, b \in \mathbb{C} \backslash\{0\}$ and $c>|a|+|b|+1$. Suppose that $f(z)=z+\sum_{n=2}^{\infty} a_{n} z^{n} \in \mathcal{R}^{t}(A, B)$. By Lemma 2.5, we need only to show that

$$
T_{4}:=\sum_{n=2}^{\infty} n(2 n-1)\left|\frac{(a)_{n-1}(b)_{n-1}}{(c)_{n-1}(1)_{n-1}} a_{n}\right| \leq 1 .
$$

Then, from (1.7) and $(|a|)_{n}=|a|(|a|)_{n-1}$, we have

$$
\begin{aligned}
T_{4} & \leq(A-B)|t|\left[\sum_{n=1}^{\infty}(2 n+1) \frac{(|a|)_{n}(|b|)_{n}}{(c)_{n}(1)_{n}}\right] \\
& =(A-B)|t|\left[2 \sum_{n=1}^{\infty} \frac{(|a|)_{n}(|b|)_{n}}{(c)_{n}(1)_{n-1}}+\sum_{n=0}^{\infty} \frac{(|a|)_{n}(|b|)_{n}}{(c)_{n}(1)_{n}}-1\right] \\
& =(A-B)|t|\left[\frac{2|a||b|}{c} \frac{\Gamma(c-|a|-|b|-1) \Gamma(c+1)}{\Gamma(c-|a|) \Gamma(c-|b|)}+\frac{\Gamma(c-|a|-|b|) \Gamma(c)}{\Gamma(c-|a|) \Gamma(c-|b|)}\right]
\end{aligned}
$$




$$
\begin{aligned}
& =(A-B)|t|\left[\frac{\Gamma(c-|a|-|b|) \Gamma(c)}{\Gamma(c-|a|) \Gamma(c-|b|)}\left(\frac{2|a b|}{c-|a|-|b|-1}+1\right)-1\right] \\
& \leq 1
\end{aligned}
$$

by (3.7), and so we have Theorem 3.6.

Theorem 3.7 Let $a, b \in \mathbb{C} \backslash\{0\}, c>|a|+|b|$ with $|a| \neq 1,|b| \neq 1$, and $c \neq 1$ and $f \in \mathcal{R}^{t}(A, B)$. Suppose that

$$
(A-B)|t|\left[\frac{\Gamma(c-|a|-|b|) \Gamma(c)}{\Gamma(c-|a|) \Gamma(c-|b|)}\left(3-\frac{2(c-|a|-|b|)}{(|a|-1)(|b|-1)}\right)+\frac{2(c-1)}{(|a|-1)(|b|-1)}-1\right] \leq 1 .
$$

Then the operator $I_{a, b ;} f$ maps $\mathcal{R}^{t}(A, B)$ into $\mathcal{U S T}$.

Proof Let $a, b \in \mathbb{C} \backslash\{0\}$ and $c>|a|+|b|$ with $|a| \neq 1,|b| \neq 1$ and $c \neq 1$. Suppose that $f(z)=$ $z+\sum_{n=2}^{\infty} a_{n} z^{n} \in \mathcal{R}^{t}(A, B)$. By Lemma 2.6, it suffices to show that

$$
T_{5}:=\sum_{n=2}^{\infty}(3 n-2)\left|\frac{(a)_{n-1}(b)_{n-1}}{(c)_{n-1}(1)_{n-1}} a_{n}\right| \leq 1
$$

Then, from (1.7) and $(|a|)_{n}=|a|(|a|)_{n-1}$, we have

$$
\begin{aligned}
T_{5} \leq & (A-B)|t|\left[3 \sum_{n=2}^{\infty} \frac{(|a|)_{n-1}(|b|)_{n-1}}{(c)_{n-1}(1)_{n-1}}-2 \sum_{n=2}^{\infty} \frac{(|a|)_{n-1}(|b|)_{n-1}}{(c)_{n-1}(1)_{n}}\right] \\
= & (A-B)|t|\left[3\left(\sum_{n=0}^{\infty} \frac{(|a|)_{n}(|b|)_{n}}{(c)_{n}(1)_{n}}-1\right)-2 \sum_{n=2}^{\infty} \frac{(c-1)(|a|-1)_{n}(|b|-1)_{n}}{(|a|-1)(|b|-1)(c-1)_{n}(1)_{n}}\right] \\
= & (A-B)|t|\left[3\left(\frac{\Gamma(c-|a|-|b|) \Gamma(c)}{\Gamma(c-|a|) \Gamma(c-|b|)}-1\right)\right. \\
& \left.-2 \frac{c-1}{(|a|-1)(|b|-1)}\left(\frac{\Gamma(c-|a|-|b|+1) \Gamma(c-1)}{\Gamma(c-|a|) \Gamma(c-|b|)}-\frac{(|a|-1)(|b|-1)}{c-1}-1\right)\right] \\
= & (A-B)|t|\left[\frac{\Gamma(c-|a|-|b|) \Gamma(c)}{\Gamma(c-|a|) \Gamma(c-|b|)}\left(3-\frac{2(c-|a|-|b|)}{(|a|-1)(|b|-1)}\right)+\frac{2(c-1)}{(|a|-1)(|b|-1)}-1\right] \\
\leq & 1,
\end{aligned}
$$

by (3.8), which completes the proof of Theorem 3.7.

Next, we give the condition on the parameters $a, b$ and $c$ that the convolution of the odd function $z F\left(a, b ; c ; z^{2}\right)$ and $f \in \mathcal{R}^{t}(A, B)$ belongs to $\mathcal{R}^{t}(A, B)$.

Theorem 3.8 Let $a, b \in \mathbb{C} \backslash\{0\}, c>|a|+|b|$ with $|a| \neq 1$ and $|b| \neq 1$ and $f \in \mathcal{R}^{t}(A, B)$. Suppose that

$$
(1+|B|)\left[\frac{\Gamma(c-|a|-|b|) \Gamma(c)}{\Gamma(c-|a|) \Gamma(c-|b|)}\left(2-\frac{c-|a|-|b|}{(|a|-1)(|b|-1)}\right)+\frac{(c-1)}{(|a|-1)(|b|-1)}-1\right] \leq 1 .
$$

Then the operator $z F\left(a, b ; c ; z^{2}\right) * f(z) \in R^{t}(A, B)$. 
Proof Let $a, b \in \mathbb{C} \backslash\{0\}$ and $c>|a|+|b|$ with $|a| \neq 1,|b| \neq 1$. Suppose that $f(z)=z+$ $\sum_{n=2}^{\infty} a_{n} z^{n} \in \mathcal{R}^{t}(A, B)$. We note that

$$
z F\left(a, b ; c ; z^{2}\right)=z+\sum_{n=2}^{\infty} \frac{(a)_{n-1}(b)_{n-1}}{(c)_{n-1}(1)_{n-1}} z^{2 n-1} .
$$

By Lemma 2.2, it is enough to show that

$$
T_{6}:=\sum_{n=2}^{\infty}(1+|B|)(2 n-1)\left|\frac{(a)_{n-1}(b)_{n-1}}{(c)_{n-1}(1)_{n-1}} a_{n}\right| \leq(A-B)|t| .
$$

Then, by a similar proof as Theorem 3.7, we get

$$
\begin{aligned}
T_{6} \leq & (A-B)|t|(1+|B|)\left[2 \sum_{n=2}^{\infty} \frac{(|a|)_{n-1}(|b|)_{n-1}}{(c)_{n-1}(1)_{n-1}}-\sum_{n=2}^{\infty} \frac{(|a|)_{n-1}(|b|)_{n-1}}{(c)_{n-1}(1)_{n}}\right] \\
= & (A-B)|t|(1+|B|)\left[\frac{\Gamma(c-|a|-|b|) \Gamma(c)}{\Gamma(c-|a|) \Gamma(c-|b|)}\left(2-\frac{c-|a|-|b|}{(|a|-1)(|b|-1)}\right)\right. \\
& \left.+\frac{(c-1)}{(|a|-1)(|b|-1)}-1\right] \\
\leq & (A-B)|t|
\end{aligned}
$$

by (3.9), and hence we have the result.

Finally, we establish the condition on the parameters $a, b$ and $c$ that the function $z F(a, b ; c ; z)$ belongs to the class $\mathcal{R}^{t}(A, B)$.

Theorem 3.9 Let $a, b \in \mathbb{C} \backslash\{0\}$ and $c>|a|+|b|+1$. Suppose that

$$
\frac{\Gamma(c-|a|-|b|) \Gamma(c)}{\Gamma(c-|a|) \Gamma(c-|b|)}\left(\frac{|a b|}{c-|a|-|b|-1}+1\right)-1 \leq \frac{(A-B)|t|}{1+|B|} .
$$

Then the function $z F(a, b ; c ; z) \in \mathcal{R}^{t}(A, B)$.

Proof By Lemma 2.2, it is sufficient to show that

$$
T_{7}:=\sum_{n=2}^{\infty}(1+|B|) n\left|\frac{(a)_{n-1}(b)_{n-1}}{(c)_{n-1}(1)_{n-1}} a_{n}\right| \leq(A-B)|t| .
$$

Then, by (ii) of Lemma 2.1, we observe that

$$
\begin{aligned}
T_{7} & \leq \sum_{n=2}^{\infty}(1+|B|) n \frac{(|a|)_{n-1}(|b|)_{n-1}}{(c)_{n-1}(1)_{n-1}} \\
& =(1+|B|)\left[\frac{\Gamma(c-|a|-|b|) \Gamma(c)}{\Gamma(c-|a|) \Gamma(c-|b|)}\left(\frac{|a b|}{c-|a|-|b|-1}+1\right)-1\right] \\
& \leq(A-B)|t|,
\end{aligned}
$$

by (3.10). This completes the proof of Theorem 3.9. 


\section{Competing interests}

The authors declare that they have no competing interests.

\section{Authors' contributions}

All authors jointly worked on the results and they read and approved the final manuscript.

\section{Acknowledgements}

Dedicated to Professor Hari M Srivastava.

The authors would like to express their thanks to the editor and the referees for many valuable advices regarding a previous version of this paper. This research was supported by the Basic Science Research Program through the National Research Foundation of Korea (NRF) funded by the Ministry of Education, Science and Technology (No. 2012-0002619).

\section{Received: 4 January 2013 Accepted: 26 March 2013 Published: 11 April 2013}

\section{References}

1. Dixit, KK, Pal, SK: On a class of univalent functions related to complex order. Indian J. Pure Appl. Math. 26, 889-896 (1995)

2. Ponusamy, S, Rønning, F: Starlikeness properties for convolutions involving hypergeometric series. Ann. Univ. Mariae Curie-Sklodowska, Sect. A 52, 141-155 (1998)

3. Dashrath: On some classes related to spiral-like univalent and multivalent functions. Ph.D. Thesis, Kanpur University, Kanpur (1984)

4. Caplinger, TR, Causey, WM: A class of univalent functions. Proc. Am. Math. Soc. 39, 357-361 (1973)

5. Padmanabhan, KS: On a certain class of functions whose derivatives have a positive real part in the unit disc. Ann. Pol. Math. 23, 73-81 (1970/71)

6. Silverman, H: Univalent functions with negative coefficients. Proc. Am. Math. Soc. 51, 109-116 (1975)

7. Goodman, AW: On uniformly convex functions. Ann. Pol. Math. 56, 87-92 (1991)

8. Goodman, AW: On uniformly starlike functions. J. Math. Anal. Appl. 155, 364-370 (1991)

9. Ponusamy, S, Vuorinen, M: Univalence and convexity properties for Gaussian hypergeometric functions. Preprint 82, Department of Mathematics, University of Helsinki, 34 pp. (1995)

10. Ruscheweyh, St, Singh, V: On the order of starlikeness of hypergeometric functions. J. Math. Anal. Appl. 113, 1-11 (1986)

11. Dziok, J, Srivastava, HM: Certain subclasses of analytic functions associated with the generalized hypergeometric function. Integral Transforms Spec. Funct. 14, 7-18 (2003)

12. Srivastava, HM, Manocha, HL: A Treatise on Generating Functions. Wiley, New York (1984)

13. Srivastava, HM: Univalent and starlike integral operators and certain associated families of linear operators. In: $L i, Z$, Ren, F, Yang, L, Zhang, S (eds.) Proceedings of the Conference on Complex Analysis, pp. 188-207. International Press, Somerville (1994)

14. Swaminathan, A: Certain sufficiency conditions on Gaussian hypergeometric functions. J. Inequal. Pure Appl. Math. 5, Article ID 83 (2004)

15. Jack, IS: Functions starlike and convex of order $\alpha$. J. Lond. Math. Soc. 3, $469-474$ (1971)

16. Subramanian, KG, Murugusundaramoorthy, G, Balasubrahmanyam, P, Silverman, H: Subclass of uniformly convex and uniformly starlike functions. Math. Jpn. 42, 512-522 (1995)

17. Duren, PL: Univalent Functions. Springer, New York (1983)

doi:10.1186/1687-1847-2013-101

Cite this article as: Kim and Cho: Properties of convolutions for hypergeometric series with univalent functions. Advances in Difference Equations 2013 2013:101.

\section{Submit your manuscript to a SpringerOpen ${ }^{\circ}$ journal and benefit from:}

- Convenient online submission

Rigorous peer review

- Immediate publication on acceptance

- Open access: articles freely available online

- High visibility within the field

- Retaining the copyright to your article 